\title{
Multi-stage learning for segmentation of aortic dissections using a prior aortic anatomy simplification
}

\author{
Duanduan Chen ${ }^{\mathrm{a}, *}$, Xuyang Zhang ${ }^{\mathrm{a}}$, Yuqian Mei ${ }^{\mathrm{a}}$, Fangzhou Liao ${ }^{\mathrm{b}}$, Huanming Xu ${ }^{\mathrm{a}}$, Zhenfeng Li ${ }^{\mathrm{a}}$, Qianjiang Xiao ${ }^{\mathrm{c}}$, Wei Guo ${ }^{\mathrm{d}}$, \\ Hongkun Zhang ${ }^{\mathrm{e}}$, Tianyi Yan ${ }^{\mathrm{a}, *}$, Jiang Xiong, ${ }^{\mathrm{d}, *}$, Yiannis Ventikos ${ }^{\mathrm{f}, \mathrm{a}}$
}

${ }^{a}$ School of Life Science, Beijing Institute of Technology, Beijing, China

${ }^{b}$ Institute of Information Engineering, Chinese Academy of Sciences, Beijing, China

${ }^{c}$ Shukun (Beijing) Network Technology Co. Ltd., Beijing, China

${ }^{d}$ Department of Vascular and Endovascular Surgery, Chinese PLA General Hospital, Beijing, China

${ }^{e}$ Department of Vascular Surgery, First Affiliated Hospital of Medical College, Zhejiang University, Hangzhou, China

${ }^{f}$ Department of Mechanical Engineering, University College London, London, UK

\section{A R T ICLE INFO}

\section{Article history:}

Received -

Received in final form -

Accepted -

Available online -

Communicated by -

2000 MSC: 41A05, 41A10, 65D05, $65 \mathrm{D} 17$

Keywords: Deep learning, Aortic dissection, Segmentation, CT-angiography, Prior anatomy simplification

\begin{abstract}
A B S T R A C T
Aortic dissection (AD) is a life-threatening cardiovascular disease with a high mortality rate. The accurate and generalized 3-D reconstruction of AD from CT-angiography can effectively assist clinical procedures and surgery plans, however, is clinically unavaliable due to the lacking of efficient tools. In this study, we presented a novel multi-stage segmentation framework for type B AD to extract true lumen (TL), the false lumen (FL) and all branches (BR) as different classes. Two cascaded neural networks were used to segment the aortic trunk and branches and to separate the dual lumen, respectively. An aortic straightening method was designed based on the prior vascular anatomy of AD, simplifying the curved aortic shape before the second network. The straightening-based method achieved the mean Dice scores of 0.96, 0.95 and 0.89 for TL, FL, and BR on a multi-center dataset involving 120 patients, outperforming the end-to-end multi-class methods and the multi-stage methods without straightening on the dual-lumen segmentation, even using different network architectures. Both the global volumetric feature of the aorta and the local characteristics of the primary tear could be better identified and quantified based on the straightening. Comparing to previous deep learning methods dealing with $\mathrm{AD}$ segmentations, the proposed framework presented advantages in segmentation accuracy.
\end{abstract}

(C) 2020 Elsevier B. V. All rights reserved.

\section{Introduction}

Aortic dissection (AD) is a serious cardiovascular disease. It is initiated by a tear on the aortic intima and followed with a surge of blood flowing into the aortic wall, splitting the original single lumen of the aorta into a true (TL) and false lumen

\footnotetext{
${ }^{*}$ Corresponding authors.
}

e-mail: duanduan@bit.edu.cn (Duanduan Chen), yantianyi@bit.edu.cn (Tianyi Yan), xiongjiangdoc@126.com (Jiang Xiong)
(FL) (Criado, 2011, Hagan et al., 2000)(Figure 1). Due to the FL development, the disease may cause insufficient blood supply to vital organs and/or induce rupture of the aorta (Criado. 2011; Nienaber and Clough, 2015). Based on the dissectionaffected region, the disease is commonly categorized as Stanford type A and B, where type A dissections affect the ascending aorta while that in type B only affects the descending aorta or beyond (White et al., 2013). Type A AD is markedly lethal and often requires urgent open surgery to replace the ascending aorta (Khan and Nair, 2002, Tsai et al., 2009), while 


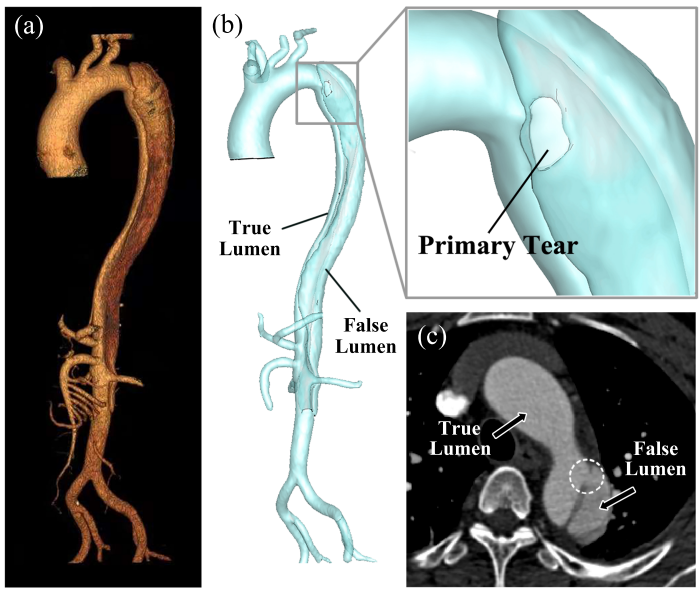

Fig. 1. Aortic dissection in 3-D/2-D views. (a) The 3-D AD extracted from CT workstation. (b) Reconstruction of AD from simulation software based on the manual labeling of CT-angiography, with the primary tear shown in the box. (c) The TL and FL in an axial slice with the primary tear shown in the dashed circle.

type $\mathrm{B} A \mathrm{~A}$ allows for more time and is commonly treated with medication or thoracic endovascular aortic repair (Chen et al. 2013: Khan and Nair. 2002). The diagnosis, risk evaluation and surgery/intervention planning of AD all rely on medical imaging, in which CT-angiography is the most common imaging modality. Morphological indicators including aortic diameter, luminal volume, position and size of tears are important for determining the necessity of surgery/intervention and indispensable for personalized treatment planning. Traditional morphological analyses by CT workstations, although presenting 3-D rendering of the contrast-enhanced vessels based on threshold, mainly support routine 2-D operations. Measurements without a mastery of global structure are empirical. Computational hemodynamics is increasingly used in analyzing cardiovascular disease (Chen et al., 2013; Xu et al., 2020), since it provides functional characteristics of the disease and assists clinical strategy. However, these analyses are also highly dependent on patient-specific aortic models, which are manually segmented and reconstructed by image processing software. This process is time-consuming, may conflict with clinical protocols and time constraints and may involve personal bias. Thus, to avoid 3-D structural information loss, an automatic and standardized segmentation method, which supports various 3-D operations and accurate measurements, is greatly needed by both the clinical and biomedical engineering fields.

Similar to the identification or segmentation of other lesions in coronary (Wolterink et al. 2016) and cerebral arteries (Livne et al. 2019), pathological aortic structures also present challenges in this task. Most of the previous studies concentrated on enhancing the vessel structures (Frangi et al. 2000) or segmenting the healthy aorta (He et al. 2020; Morais et al. 2017, Noothout et al., 2018; Trullo et al. 2017), with only few methods reported for AD. Some of the AD-related work achieved the identification of type B AD from original images (Dehghan et al. 2017) or (semi-) automatically extracting dissection walls (or flaps) (Krissian et al., 2013; Morariu et al., 2016), while they did not segment the vascular structure. For the segmenta- tion of type $\mathrm{B} A \mathrm{AD}$, several strategies were proposed by using deformable model (Fetnaci et al., 2013), Hough transformation (Kovács et al. 2006), spatial continuity prior model (Duan et al. 2016), multi-scale wavelet analysis (Lee et al., 2008) and boundary cost minimization combined with image denoising (Fitria et al. 2019). These methods have made meaningful explorations and achieved good segmentation performances for $\mathrm{AD}$ based on a relatively small number of datasets. However, they were rule-based, requiring hand-crafted feature selection for the initialization or predominantly relying on manual processing. Moreover, these methods have not been widely used in clinic, probably due to the lack of detailed 3-D measurements and of verification being insufficient. Recently, a multistage AD segmentation framework was proposed by using deep learning (DL), aiming at extracting TL and FL and calculating the dual-lumen volumes with a fully automatic method ( $\mathrm{CaO}$ et al. 2019). However, this framework did not remove the bottleneck of the dual-lumen segmentation or evaluate the performance on identifying primary tears. Moreover, it did not account for the branch vessels (BR) of the aorta, which are important anatomical sites for both clinical evaluations and hemodynamics modelling.

DL is a subfield of machine learning, which has been rapidly developed and used in medical image processing. Convolutional neural networks $(\mathrm{CNN})$, a variant of traditional artificial neural networks, which was initially used for classification (Anthimopoulos et al. 2016; LeCun et al. 1990), has been widely applied to pixel-wise image segmentation tasks (Khened et al. 2018; Lu et al. 2019, Shelhamer et al. 2016). In the field of cardiovascular imaging, several efforts have been made to process CT-angiography via CNN models, such as vascular structures segmentation (Chen et al. 2018c), thrombus extraction (López-Linares et al., 2018) and lesion detection and classification (Zreik et al., 2018). In contrast to the rule-based nonneural-network attempts, DL-based methods avoided manual operations. The outstanding inherent capability of extracting relevant image features during the training phase created broad consensus that DL frameworks constituted the state-of-the-art performers for such applications. In this study, we proposed a new DL-based framework for segmenting type $\mathrm{B} A D$ in a multi-stage manner. Two cascaded CNN models were used: $\mathrm{CNN}_{1}$ segmented the aortic trunk (AO) and BR firstly, and then $\mathrm{CNN}_{2}$ separated TL and FL based on the extracted AO in the former stage. Moreover, between the two networks, an adaptive aorta straightening algorithm was designed relying on the prior vascular anatomy, which efficiently simplifies the second stage segmentation of the dual lumen.

In summary, our main contributions are summarized below:

- We designed a fully novel automatic type B AD segmentation pipeline for extracting TL, FL and BR as differen$t$ classes. It had superior automation compared with the traditional rule-based methods and achieved better performance than the previous DL-based model on the same dataset. The effect of different components in the proposed pipeline was sufficiently analyzed and discussed.

- Based on the prior anatomy of vascular structure, an aorta 


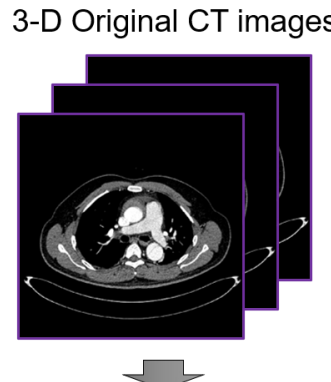

Volume of interest

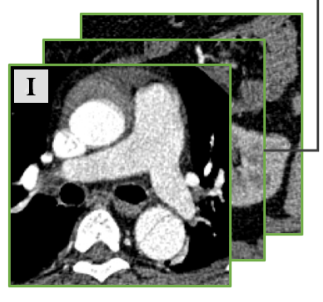

Pre-processing
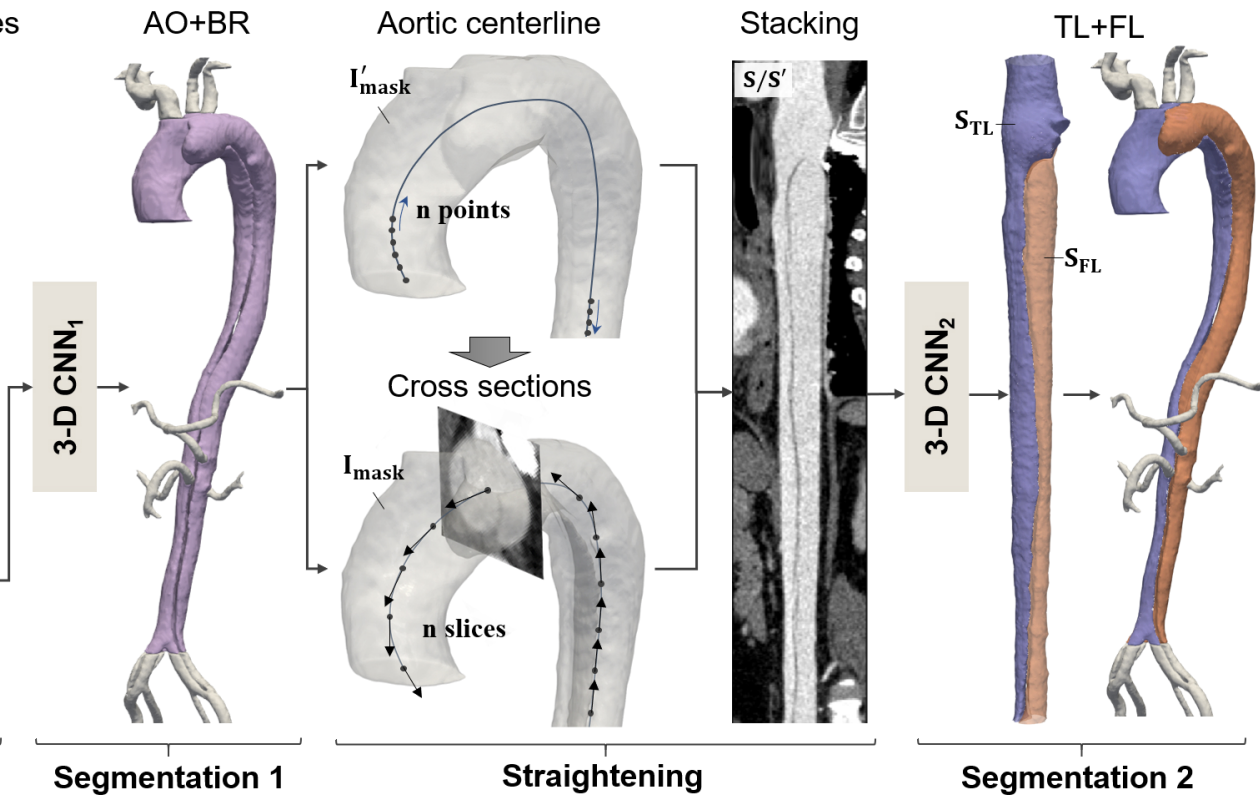

Fig. 2. The entire proposed pipeline in this study. It contained two main parts: the pre-processing of CT images and the multi-stage AD segmentation. The latter consisted of three steps: the multi-class segmentation of AO and BR (Segmentation 1), the straightening of the aorta (Straightening), and the further segmentation of the dual lumen (Segmentation 2). The key intermediate results were annotated in the pipeline (e.g., $I, I_{\text {mask }}$, and $I_{\text {mask }}^{\prime}$ ). The difference between $S$ and $S^{\prime}$ was only the $\mathrm{Z}$-axis resolution so that they had similar shape and were shown in the same figure. Note that $S^{\prime}$ was the input of $\mathrm{CNN}_{2}$.

straightening method was designed for this segmentation task, which brings a significant gain in dual-lumen segmentations and presents potential to be extensively used in other lesion vessel segmentation tasks.

- By using visualization, we discussed the difficulties encountered by applying DL methods to the scene of duallumen segmentation, and their performance on the local key morphological structure, i.e. the primary tear, thus further evaluating the clinical application value of this framework.

\section{Data}

\subsection{Patients and Image Acquisition}

This study included a multi-center dataset comprising of 120 patients (age: $53.15 \pm 11.36$ years, 97 males), which were retrospectively collected by the Chinese PLA General Hospital, the First Affiliated Hospital of Medical College of Zhejiang University, Xiangya Hospital of Central South University, Qilu Hospital of Shandong University, and the second People's Hospital of Yunnan Province. All the patients were diagnosed with Stanford type $\mathrm{B} \mathrm{AD}$ and underwent preoperative CT-angiography. Due to the various slice thickness and the difference of the scanning region, the number of the valid CT slices varied among the patient cases $(809 \pm 119.75)$. Each axial slice had $512 \times 512$ pixels. The whole aorta, internal iliac artery, and both left and right subclavian arteries were within the scanning range. These images were reconstructed with a in-plane resolution of $0.721 \pm 0.079 \mathrm{~mm}$ and slice thickness ranging from 0.5 to $1.0 \mathrm{~mm}$.

\subsection{Manual Annotation}

For training, validating and testing the proposed AD segmentation framework, all 120 scans were labeled by an expert with rich experience of reading CT images using medical image processing software Mimics 19.0 (Materialise, Leuven, Belgium). The multi-class manual annotation consisted of the background and three vascular foreground structures, i.e. TL, FL and BR, where BR comprised all branch vessels, including aortic arch branches, abdominal aortic branches and iliac arteries, and the AO was the combination of TL and FL. The whole annotation process had two stages: first, a complete mask of the AD was generated slice by slice in axial view, where some anatomical positions were defined as the endpoints of different vascular structures: ascending aorta started from the first slice upon the aortic valve; both subclavian arteries ended with the highest anatomical curvatures; renal arteries and inferior mesenteric arteries were stopped at the primary bifurcation site; celiac trunk was stopped at the secondary bifurcation site, while the remained branches were annotated till the boundary of the CT image. All the branches were separated from the aorta at the entrances. Second, the BR were removed and all the tears connecting the dual lumen were cut off to create isolated TL and FL as two another classes. The identification of tears was strictly in accordance with the criteria of the expert and no gap was left between TL and FL at tears. All scans were labeled under the same conditions, i.e. window width equal to $700 \mathrm{HU}$ and window level equal to $300 \mathrm{HU}$, avoiding irrelevant interference of visualization.

\section{Methods}

In this section, a novel AD segmentation pipeline was introduced and divided into two main parts. As shown in Figure 2 


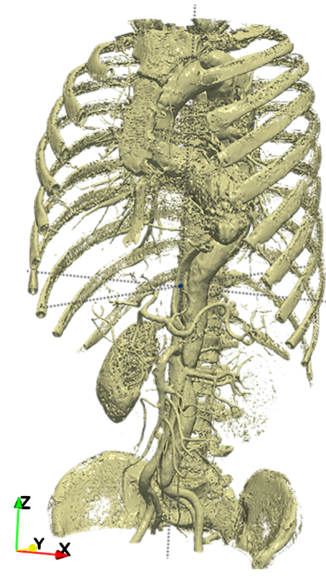

(a)

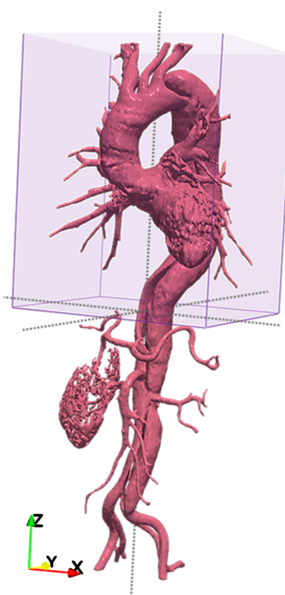

(b)

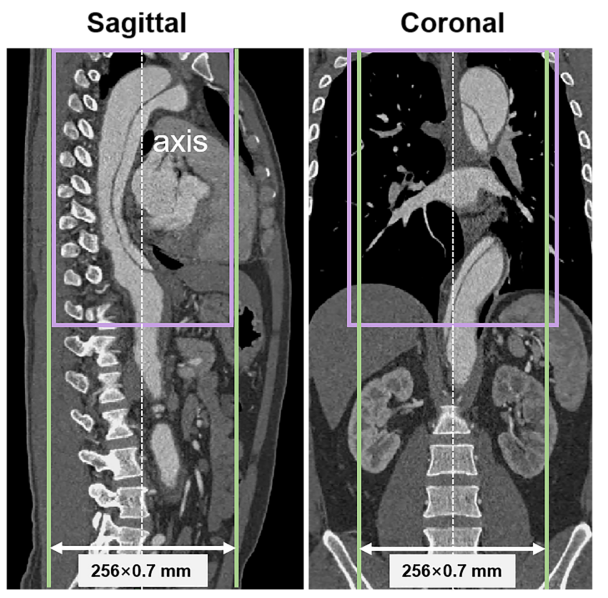

(c)

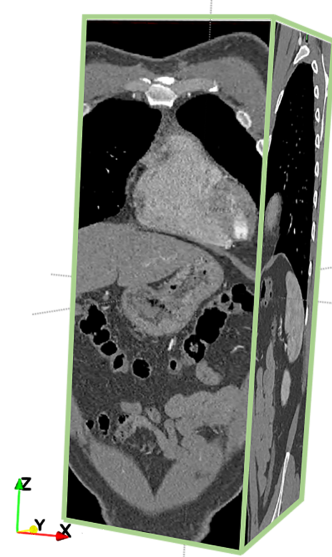

(d)

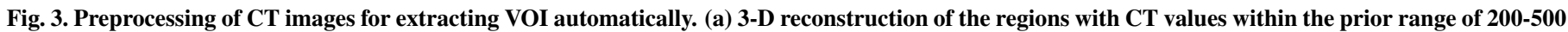

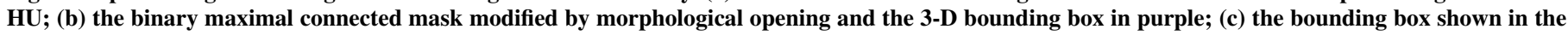

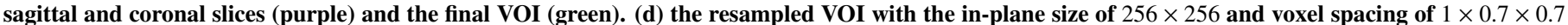
$\mathbf{m m}^{3}$.

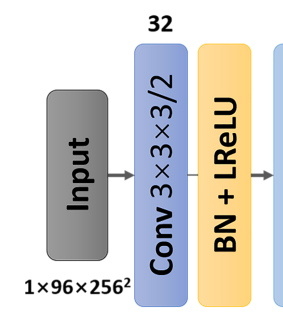

$128 \times 48 \times 128^{2}$
32

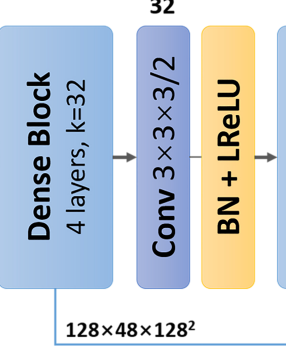

32

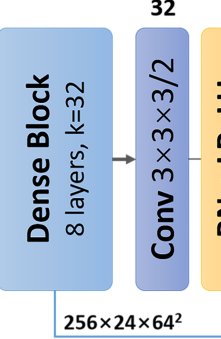

64

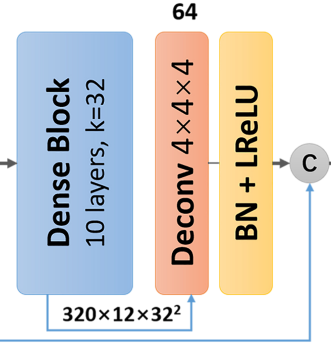

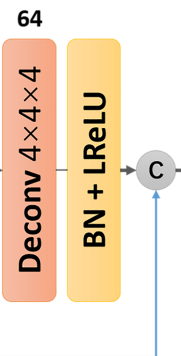

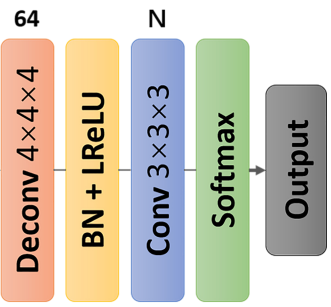

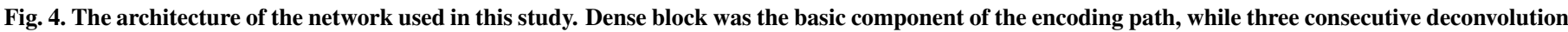

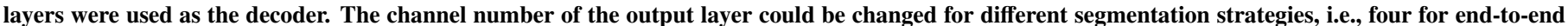
multi-class strategy and three for multi-stage $\mathrm{CNN}_{1}$ and $\mathrm{CNN}_{2}$. The input and feature map size of $\mathrm{CNN}_{1}$ were shown as examples.

it contained the preprocessing of CT images for volume of interest (VOI) extraction and multi-stage AD segmentation based on the anatomy simplification.

\subsection{Automatic VOI extraction}

The inherent shortcoming of using DL-based methods in medical 3-D scenes (Livne et al., 2019, López-Linares et al. 2018) were the large memory requirements and the resultan$\mathrm{t}$ hard-to-deploy challenges of the models in the practice (Imai et al. 2018). In CT images, AO and BR were distributed in all axial slices, so the entire CT image should be used for the network training, making the memory requirements even more severe. One strategy for alleviating this problem was to compress the original image into a smaller size by interpolation (Cao et al. 2019), however, at the expense of keeping an extended view of unrelated background and losing high resolution. In order to maintain the fine image features and simultaneously decrease the memory requirement in the training phase, we designed an intensity-based method for extracting VOI automatically.

The contrast-enhanced arteries in CT images had a prior intensity range of 200-500 HU. We extracted all the voxels within this range first and cut off the small connections using morphological opening. The maximal connected domain was subse- quently extracted as a mask for roughly locating the enhanced organs containing blood, like aorta and heart. A bounding box of the mask was generated in the coordinate system of Figure 3 . whose axis was also set as the one of VOI. For avoiding the influence of the kidneys, only the higher half part of the mask was used for generating the bounding box. We empirically specified an X-Y square region as the in-plane range of the VOI with a side length of $256 \times 0.7 \mathrm{~mm}$ in the physical world, where the 256 represented the number of resampled voxels and $0.7 \mathrm{~mm}$ represented the resampled spacing. All slices in Z-axis were included in the VOI, and resampled with the same spacing of 1.0 $\mathrm{mm}$. Hence, after the image preprocessing, all VOI from different $\mathrm{CT}$ images had the same spacing and image size in $\mathrm{X}-\mathrm{Y}$ plane, but inconsistent slices in the Z-axis due to the different scanning lengths.

\subsection{Multi-stage segmentation}

\subsubsection{Segmentation of $A O$ and $B R$}

Different from the previous DL-based method (Cao et al. 2019) feeding the network with the compressed entire CT image, we used the cropped subvolumes of VOI to train the network. It was similar to the strategies that generated image patches for segmentations (Hou et al., 2016; Nazeri et al. 2018), which also achieved good performance in practice. In 
this work, all the subvolumes were randomly extracted from the VOI with a fixed number of layers along the Z-axis and included all the in-plane voxels with the size of $256 \times 256$. All the subvolumes were fed into $\mathrm{CNN}_{1}$ for a multi-class segmentation of $\mathrm{AO}, \mathrm{BR}$, and background. We made full use of the GPU memory in the training phase to increase the subvolume layers as much as possible for providing more Z-axis features. To better learn the local image features of the subvolumes, we used dense block (Huang et al., 2017) as the basic component to construct the network, whose dense connections significantly increase the efficiency of using parameters and improve flow of information and gradients throughout the network (Gibson et al., 2018; Khened et al., 2018). As shown in Figure 4, 3 dense blocks were applied to the encoding part of the network, consisting of various numbers of layers. A $3 \times 3 \times 3$ convolutional downsample layer with stride 2 was added before each block. In the decoding part, 3 consecutive deconvolution layers were responsible for recovering the size of images, where the latter two integrated the high-resolution features with the same size from the shortcut connections. Finally, a $3 \times 3 \times 3$ convolution was used to convert multiple channels to the 3 -channel output, corresponding to three different classes. After each convolution, we used the In-Place activated batchnorm algorithm (Rota Bulò et al. 2018) to free the additional memory by avoiding the buffers needed for the gradient computation during the backward pass. For feature activation, Leaky ReLU (LReLU) (Maas et al. 2013) was used with a slope of 0.01 .

For effectively alleviating the class imbalance problem caused by the voxel number difference between background and different vascular structures, $\mathrm{CNN}_{1}$ was trained with voxel-wise multi-class dice loss (Yang et al., 2018):

$L_{1}=-\frac{N}{2} \sum_{C=1}^{N} \frac{\left\|Y_{C} \cdot \hat{Y}_{C}\right\|^{2}}{\left\|Y_{C}\right\|^{2}+\left\|\hat{Y}_{C}\right\|^{2}}$

where $Y_{C}$ and $\hat{Y}_{C}$ were the $C^{\text {th }}$ channel of the label and the output, respectively. $N$ meant the number of classes and was 3 in this design. During the training phase of this network, randomly cropped subvolumes had the size of $96 \times 256 \times 256$. For the inference, the extracted VOI was split into non-overlapping subvolumes from bottom to top with the same size and fed into the trained network. If the Z-axis length of VOI was not divisible by 96 , only the last two subvolumes could partially overlap. After the segmentation, all the subvolumes were finally concatenated based on the correct order in Z-axis, forming the entire segmentation result of VOI, which was then interpolated to the initial resolution of CT images and mapped to the original position.

\subsubsection{Straightening of the aorta}

For the AD segmentation, the separation of TL and FL was the most difficult part and might be influenced by the complex torsion and the curved shape of the aorta. In this section, we proposed to straighten the naturally curved aorta for simplifying the prior shape and eliminating the interference above before the dual-lumen segmentation.

The aorta straightening contained two steps, i.e., centerline calculation and coordinate transformation. In the first step, the maximal connected domain of the segmented $\mathrm{AO}$ from $\mathrm{CNN}_{1}$ was used as a binary mask. Both the mask and the original CT image were resampled to have isotropic resolution, which were denoted with $I_{\text {mask }}$ and $I$ (the key intermediate results were marked in Figure 2,), respectively. After that, the flap (a gap shown as background) between the dual lumen in $I_{\text {mask }}$ was filled using morphology closing, forming $I_{\text {mask }}^{\prime}$. The aortic skeleton was then calculated from $I_{\text {mask }}^{\prime}$ by skeletonization (Lee et al., 1994). The longest path of the skeleton was extracted using the Dijkstra's algorithm and set as the initial centerline of the whole aorta. Due to the skeletal points having step-wise coordinates, which could not carry the true gradient information of the centerline, we designed a new adaptive method for adjusting the coordinates, i.e., iteratively modifying a displacement vector for each point. In this design, three types of losses were derived and combined to guide the convergence of the algorithm. Firstly, we applied distance transformation to $I_{\text {mask }}^{\prime}$ and the output values were normalized. During each iteration, the centerline was moved using the recent displacement vectors and the result was denoted with coordinate vector $c$. The values on the points of $c$ were trilinearly interpolated based on the normalized values above, forming a value vector $c_{v}$. The first part of the loss was described as:

$l_{1}\left(c_{v}\right)=\frac{1}{n} \sum_{i=1}^{n} \tan \frac{\left(1-c_{v}^{i}\right) \pi}{2}$

where $n$ represents the number of points on the centerline and $c_{v}^{i}$ denotes the $i^{\text {th }}$ point value. This loss could constraint the point movement by decreasing the displacement magnitude, making the line near the center of the aorta. In order to keep the similar distances between the adjacent points, we denoted $d^{j}=\| c^{j+1}$ $c^{j} \|$, where $c^{j}$ represents the coordinate of the $j^{\text {th }}$ point, and the second loss was as follow:

$l_{2}(c)=\frac{1}{n-1} \sum_{j=1}^{n-1}\left(d^{j}-\bar{d}\right)^{2}$

where $\bar{d}=\sum_{j=1}^{n-1} d^{j} /(n-1)$. Last but not least, the smoothness of the centerline was improved by the third part of the loss:

$l_{3}(c, \delta)=\frac{1}{n-2 \delta} \sum_{k=1}^{n-2 \delta}\left\|c^{k+2 \delta}+c^{k}-2 c^{k+\delta}\right\|^{2}$

where $\delta \in\{1,2, \ldots,\lfloor(n-1) / 2\rfloor\}$ and was the half interval influenced by each point. In this study, $\delta=1,2,3$ was used. All above losses were combined in the following form:

$l_{\text {line }}\left(c, c_{v}\right)=l_{1}\left(c_{v}\right)+\beta l_{2}(c)+\omega \sum_{\delta} l_{3}(c, \delta)$

and optimized using gradient descent with the learning rate of 1. Only when the change of the loss was smaller than $10^{-7}$, the iteration was stopped. In this study, the scalars $\beta$ and $\omega$ were both 0.1 .

In the second step, i.e., coordinate transformation, we firstly initialized local 2-D coordinate systems based on the modified centerline. Each coordinate system centered on a point of the 
line and was in the plane of the corresponding aortic cross section. Therefore, a total $\mathrm{n}$ local systems were constructed. For the initialization of each coordinate system, the in-plane $\mathrm{X}$ and $\mathrm{Y}$ axis were denoted with an unit vector $x$ and $y$, respectively, which were randomly selected but orthometric in each plane. All above coordinate systems should be finely rotated for improving the smoothness between the adjacent planes. In this study, we denoted the rotation angle with $\theta$ and the axis after the rotation with $v_{\theta}=\left\{x_{\theta}, y_{\theta}\right\}$. The iteration for optimizing $\theta$ used the loss as below:

$l_{\text {plane }}\left(v_{\theta}\right)=\frac{1}{n-2 \delta^{\prime}} \sum_{v \in v_{\theta}} \sum_{\delta^{\prime}} \sum_{m=1}^{n-2 \delta^{\prime}}\left\|v^{m+2 \delta^{\prime}}+v^{m}-2 v^{m+\delta^{\prime}}\right\|^{2}$

where $\delta^{\prime} \in\{1,2, \ldots,\lfloor(n-1) / 2\rfloor\}$ and had the values of $\{1,2,3\}$. Gradient descent was also used with learning rate of 1 in our setting. After the two-step centerline optimization, new cross sections could be resampled from $I$ on the 2-D local coordinate systems and finally stacked up as the straightened image $S$. Each resampled cross section had the same space resolution with $I$, and the size was $96 \times 96$. All the values of $S$ were calculated using trilinear interpolation.

\subsubsection{Separation of $T L$ and $F L$}

In this stage, we designed the second network $\mathrm{CNN}_{2}$ for the segmentation of TL and FL. In order to better capture the global dual-lumen features like lengths and volumes, we trained $\mathrm{CNN}_{2}$ with the entire straightened aorta in $S$, which should be first resampled as $S^{\prime}$ with the same image size of $640 \times 96 \times 96$. $\mathrm{CNN}_{2}$ had the same main architecture with $\mathrm{CNN}_{1}$ but output the probability maps of the straightened TL and FL simultaneously. The multi-class dice loss was also used for the training phase. For inference, we used the image straightened based on AO output by $\mathrm{CNN}_{1}$ rather than the annotation, directly evaluating the performance of the whole framework on the dual-lumen segmentation.

\subsubsection{Morphological recovery}

During the aorta straightening, the mapping relationship of each couple of $I$ and $S$ was saved in a matrix. We could use this matrix to recover the curved shape of the segmented TL and FL. In our design, the voxel nearest to an interpolated point in the original space was inversely filled with the corresponding prediction in the straightening space. However, notably it was a preliminary recovery process because the curved dual lumen were very loose with a lot of holes inside. We subsequently filled every hole voxel with the value of the nearest predicted vascular voxel. Finally, the resolution of the compact TL and FL were recovered to the original value of CT images by using the nearest neighbor interpolation.

\subsection{Data augmentation}

The training of $\mathrm{CNN}_{2}$ with the entire aorta caused a severe reduction of the training samples. Data augmentation was adopted to improve the segmentation performance, i.e., each straightened image $S^{\prime}$ was rotated with a random angle and scaled with a factor in the range of 0.75-1.25. The augmentation was only carried out in the X-Y plane. Moreover, in the first segmentation step, we did not use any augmentation methods.

\section{Experiments and results}

In this section, we described the experimental set up in detail, including the implemental configurations, evaluation metrics, and the specific experiments. The straightening performance of the $\mathrm{AD}$ was firstly shown in section 4.2. In order to prove the advantage of the proposed framework, the comparisons with an end-to-end multi-class segmentation strategy and the multistage strategies without straightening were presented in section 4.3 The quantitative and qualitative analysis of the morphological results were performed in section 4.4 Finally, we analyzed the influence of the network by applying various architectures to different strategies in section 4.5

\subsection{Implementation and metrics}

For adequately evaluating the proposed framework, we adopted 6-fold cross validation on the 120 patients, i.e., each training set had $80 \mathrm{CT}$ images, while the validation and testing set had 20 images. For evaluating TL and FL of the testing set in each fold, two networks with the best validation performance in the corresponding segmentation stages were embedded in the pipeline which was tested. Note that the chosen models were trained based on the same fold. In single epoch of the training phase, each VOI was randomly cropped 50 times for $\mathrm{CNN}_{1}$, while each straightened image was sampled 30 times for $\mathrm{CNN}_{2}$ using augmentation. All networks were trained from scratch using 4 NVIDIA GeForce GTX 1080Ti GPUs synchronously with a batch size of 4 , and optimized by stochastic gradien$t$ descent with the momentum of 0.9 . The initial learning rate was 0.01 and divided by 10 every 50 epochs. The weight decay was set to 0.0001 . Convergence was defined as a state in which no substantial progress was observed in the validation dice score. About 80,000 iterations were performed for training $\mathrm{CNN}_{1}$, while 40,000 for $\mathrm{CNN}_{2}$. All experiments were carried out using Python programming language, while the network$\mathrm{s}$ were constructed and trained based on the Pytorch library (Paszke et al., 2017).

Three metrics were used to compare the performances: dice similarity coefficient (DSC), mean boundary distance (MBD), and symmetric $95 \%$ Hausdorff distance $\left(\mathrm{HD}_{95}\right)$ of the boundary:

$$
\begin{aligned}
D S C & =\frac{2|R \cap G|}{|R|+|G|} \\
M B D & =\max (\overline{D(R, G)}+\overline{D(G, R)}) \\
H D_{95} & =\frac{P_{95}[D(R, G)]+P_{95}[D(G, R)]}{2}
\end{aligned}
$$

where $R$ and $G$ are the segmentation result and the ground truth. $D(R, G)$ is the set of distances from boundary voxels of $R$ to the nearest boundary voxel of $G . P_{95}[D]$ means the $95^{\text {th }}$ percentile of $D$.

\subsection{The performance of straightening}

In order to evaluate the aorta straightening method, we drew the loss curves of the two smoothing steps (i.e., the centerline smoothing and plane rotating) in Figure 5(a). All the centerlines 
(a)

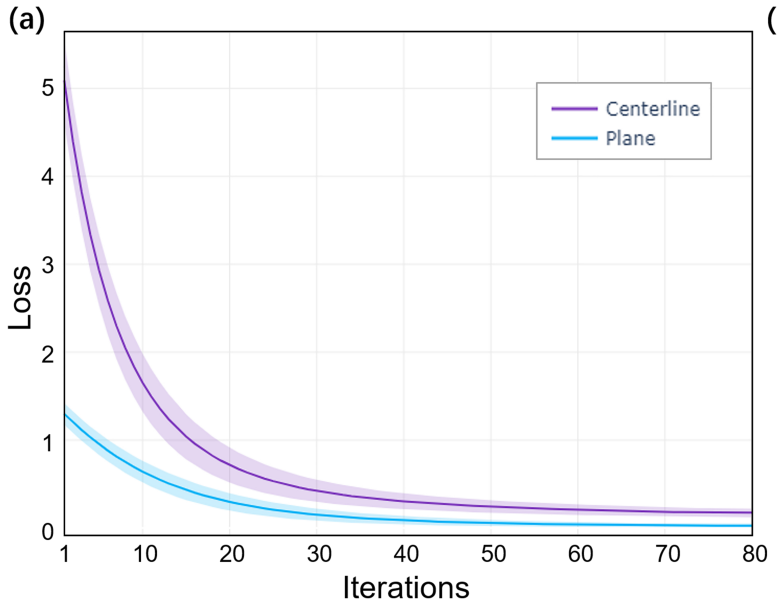

(b)

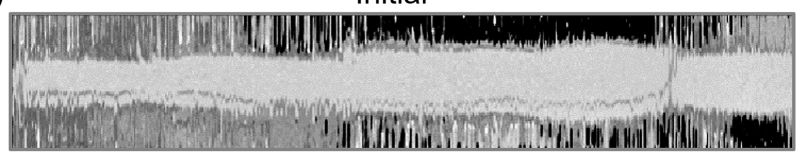

Step 1 (Centerline smoothing)

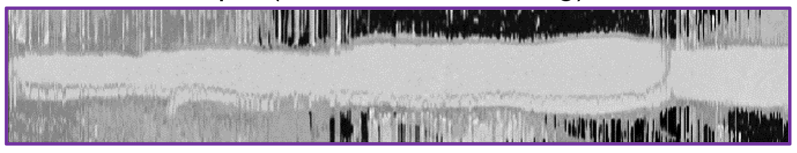

Step 2 (Plane rotating)

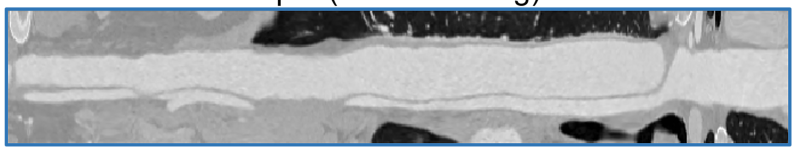

Fig. 5. The performance on aorta straightening using $\beta, \omega=0.1$. (a) Loss curves in two smoothing steps. The shaded areas represented the standard deviation of the loss. (b) The visualization of one case in different steps, i.e., the initial straightening without any smoothing (first), the result after centerline smoothing (second), and the final result after plane rotating (third).
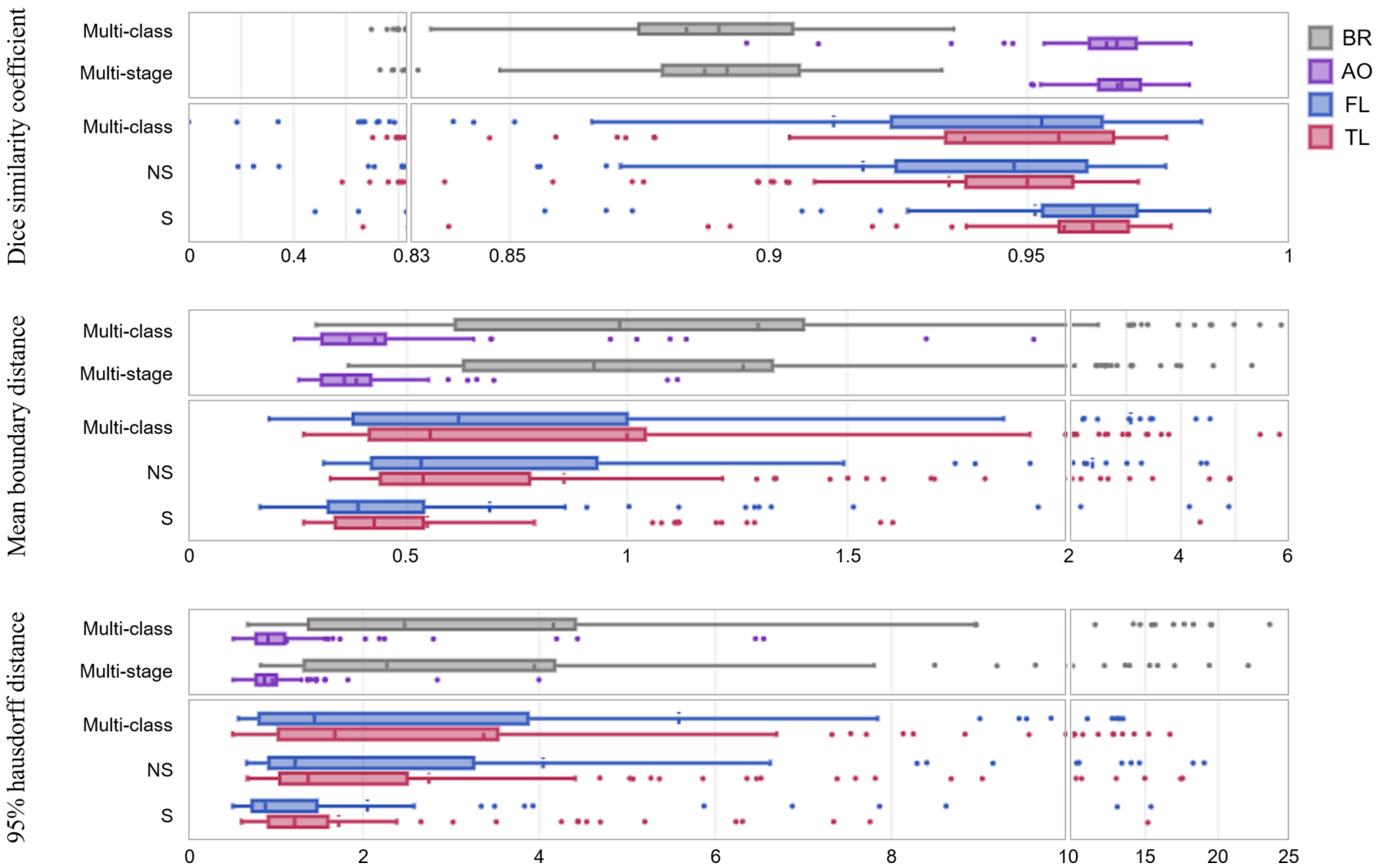

Fig. 6. Boxplots showing segmentation performances of different strategies.

were extracted based on the result $\mathrm{AO}$ of the first segmentation stage. The visualized result of one case in different steps were shown in Figure 5(b). It was obvious that the smoothing efficiently improved the straightening performance, where the borders of the dual lumen and the flap had better continuity. Additionally, a group of experiments were designed for evaluating the influence of scalars (i.e., $\beta$ and $\omega$ in Eq.(5p) on the final dual-lumen segmentation. The results were presented in Appendix and we found that there was no obvious trend shown in the comparisons. The used configuration $(\beta, \omega=0.1)$ just resulted in slightly higher dice score.

\subsection{The effect of segmentation strategies}

In this study, several modules assembled in the proposed framework should be further evaluated for confirming their contributions to this task. Therefore, we designed two strategies for a horizontal comparison. Firstly, an end-to-end multi-class segmentation method was set as the baseline in this design, which used a single network with the same architecture as $\mathrm{CNN}_{1}$ to segment TL, FL, and BR simultaneously. Considering the limitation of the GPU memory and the large CT image size with high resolution, we trained the network with the cropped subvolumes of $\mathrm{VOI}$ and the same settings for $\mathrm{CNN}_{1}$ to approximate 


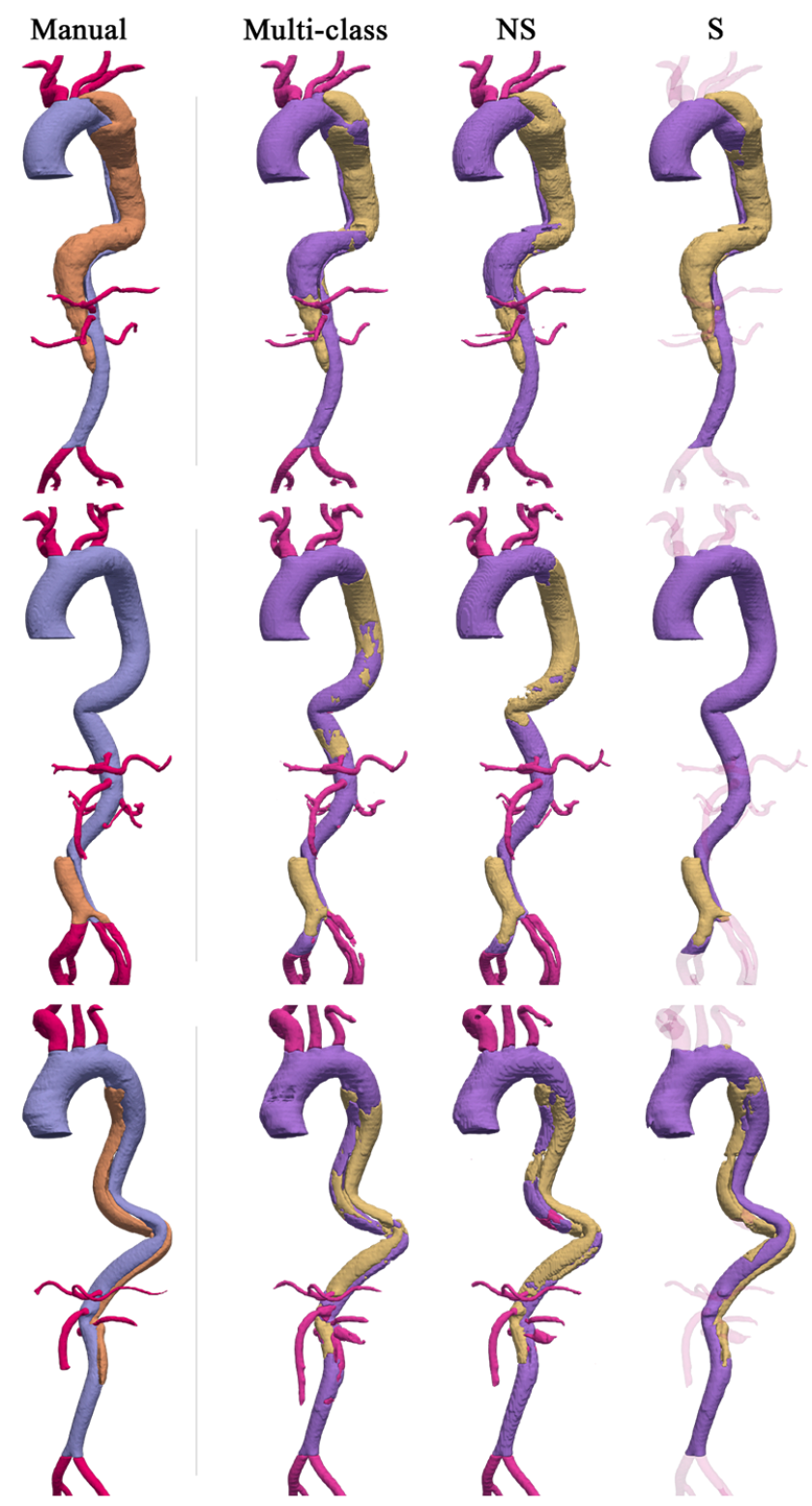

Fig. 7. 3-D reconstructions of the segmentations based on different strategies. BR of NS and S were the same, so only the one in NS was shown fully opaquely for a better comparison of the dual lumen.

the end-to-end design. Moreover, we removed the straighting step and used only two cascaded networks to achieve the segmentation (abbreviated as NS). The $\mathrm{CNN}_{2}$ was fed with the image region only containing $\mathrm{AO}$, which was extracted based on $I_{\text {mask }}$. Meanwhile, the proposed straightening-based framework was abbreviated as $\mathrm{S}$. All $\mathrm{CNN}_{2}$ in the multi-stage pipelines used the same network in section 3.2.3 and were trained with the same settings in section 4.1. All strategies were evaluated based on 6-fold cross validation. The results of 6 testing sets were combined for statistical analysis.

The boxplots of the horizontal comparison were shown in Figure 6. To evaluate the results of $\mathrm{AO}$ and $\mathrm{BR}$, the multi-class results of TL and FL were also combined to form the AO compared with the one output by the multi-stage framework. The multi-stage strategies performed slightly better both on $\mathrm{AO}$ and $\mathrm{BR}$, and produced fewer outliers on AO. For the segmentation
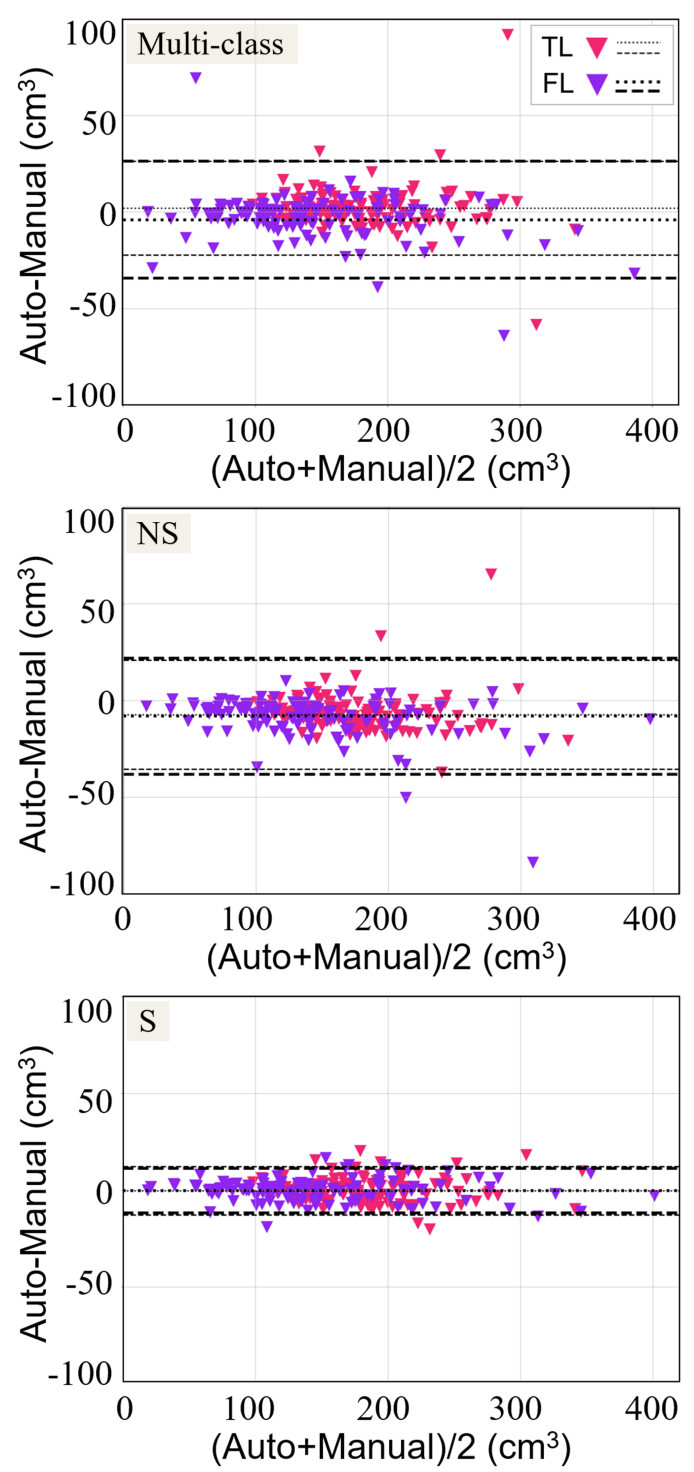

Fig. 8. Bland-Altman analysis of TL and FL volumes of end-to-end multiclass and two multi-stage strategies with manual quantification.

of the dual lumen, the straightening-based method was obviously better than the NS-based and the multi-class methods. 3-D reconstructions of the segmentations were visualized in Figure 7. Because the BR of multi-stage methods were the same, we only set the opacity of BR in NS as 1, making it easier to compare the morphology of the dual lumen.

\subsection{Morphological analysis}

\subsubsection{Quantitative analysis of lumen volumes}

In clinical practice, the volume of the dual lumen was one of the most essential morphological characteristics, which could assist the individual surgical planning and be useful for analyzing the surgical outcomes. We calculated the volumes of the segmented TL and FL for evaluating the practicality of our framework. The multi-class and two multi-stage strategies were all compared to the manual quantification by using BlandAltman analysis with the results shown in Figure 8. 
3-D
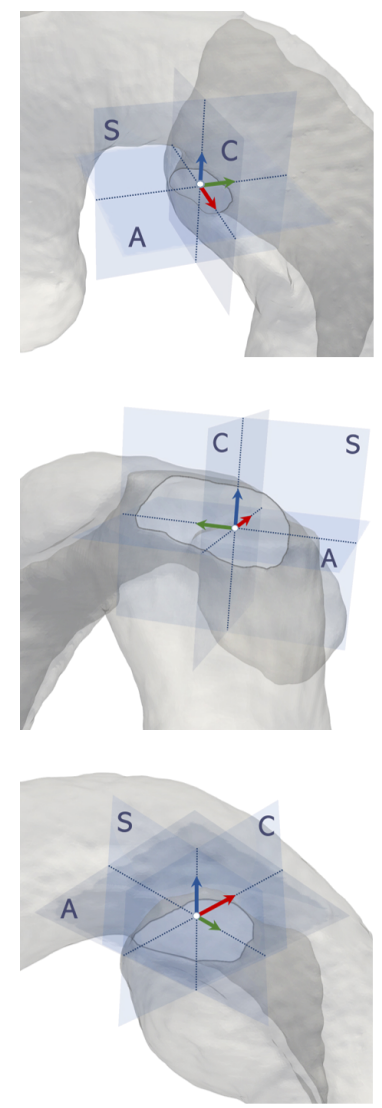

Axial
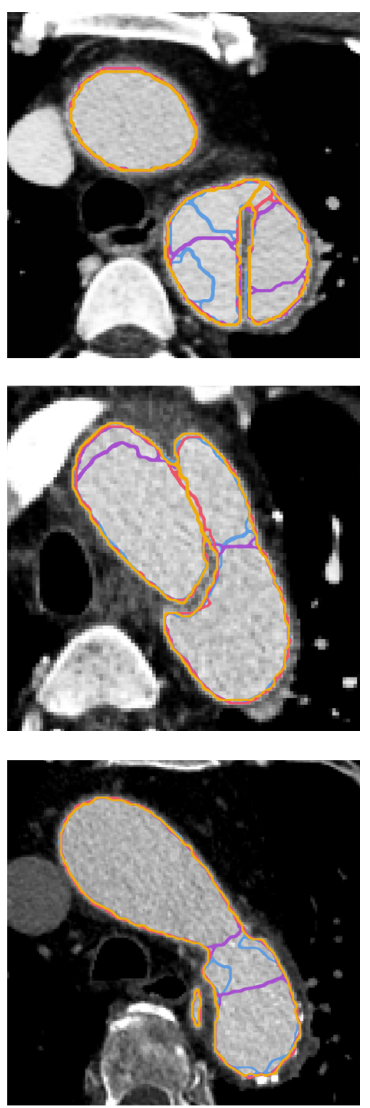

Manual
Sagittal
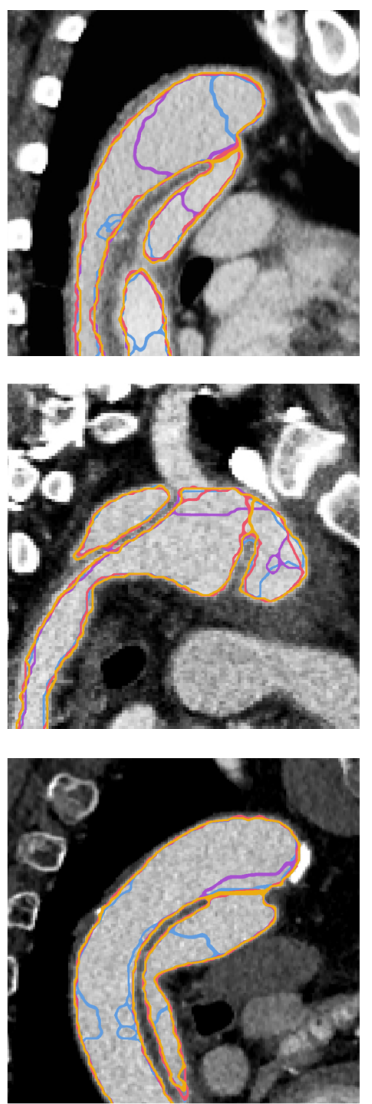

NS
Coronal
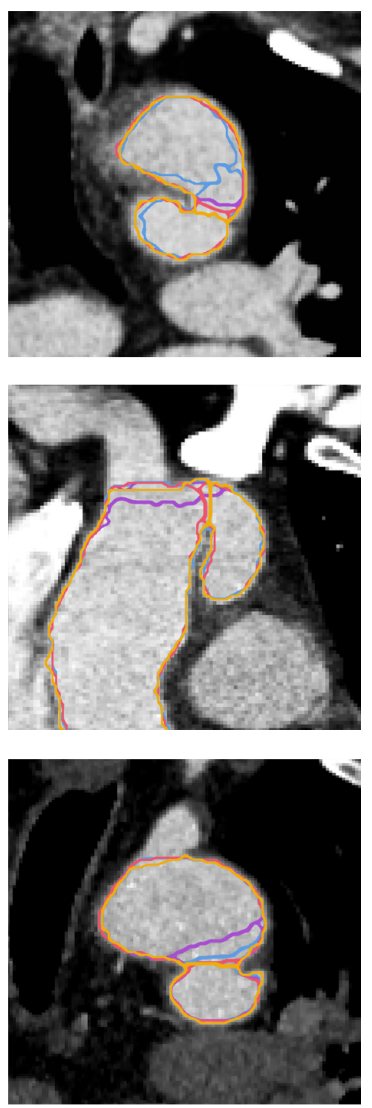

S

Fig. 9. The performances on primary tears of three patients. The first column showed the local 3-D reconstructions near the primary tear. A, S, and C represented axial, sagittal, and coronal planes, respectively. The boundary of the segmentations based on different strategies were delineated and compared in the different views. The lines inside the contrast-enhanced regions represented the interfaces of TL and FL, which were also recognized as primary tears.

\subsubsection{Qualitative analysis of tears}

For the separation of the dual lumen in preoperative CT images, one of the most difficult tasks was grasping the distinction of TL and FL locally at the aortic arch (Fetnaci et al., 2013, Kovács et al., 2006). We qualitatively compared the local segmentation results by visualizing the performances on identifying primary tears, which have been delineated as the interfaces between the dual lumen in Figure 9. Similarly, the multi-class and two multi-stage methods were tested. It was obvious that the boundary of the strategy $\mathrm{S}$ was smoother and more similar to the ground truth than the others.

\subsection{The effect of CNN networks}

For further understanding the influence of the CNN architectures on this segmentation task, we chose three other mature models, i.e., 3D U-Net (Çiçek et al., 2016), SegNet (Badrinarayanan et al. 2017), and DeepLabV3 + (Chen et al., 2018b), to replace the network used in the proposed framework. In this study, the end-to-end multi-class network, the multi-stage $\mathrm{CNN}_{1}$, and $\mathrm{CNN}_{2}$ of $\mathrm{NS}$ and $\mathrm{S}$ were all replaced. 6-fold cross validation was used and all the testing sets were combined to show the performances of these different strategies. Details of
Table 1. The properties of the tested CNN architectures. For each network, the following were reported: the maximum acceptable slice number of the cropped subvolume from VOI for training multi-class model and $\mathrm{CNN}_{1}$ (\#Slices); the number of trainable weights (\#Weights); average and SD inference time per case for $\mathrm{CNN}_{1}\left(\right.$ Time $\left._{1}\right)$ and $\mathrm{CNN}_{2}\left(\right.$ Time $\left._{2}\right)$.

\begin{tabular}{lllll}
\hline & \#Slices & \#Weights(M) & Time $_{1}(\mathrm{~s})$ & Time $_{2}(\mathrm{~s})$ \\
\hline 3D U-Net & 96 & 16.82 & $13.10 \pm 1.67$ & $0.93 \pm 0.02$ \\
SegNet & 128 & 46.85 & $10.50 \pm 1.59$ & $0.66 \pm 0.04$ \\
DeepLabV3+ & 192 & 132.93 & $10.52 \pm 1.90$ & $0.55 \pm 0.12$ \\
Ours & 96 & 6.48 & $14.19 \pm 2.15$ & $0.91 \pm 0.03$ \\
\hline
\end{tabular}

the networks and experimental settings were presented in Table 1. Both the training of the multi-class network and $\mathrm{CNN}_{1}$ made full use of the GPU memory by using maximum acceptable number of the slices in the subvolumes. The results of the testing sets were presented in Table 2, showing the comparison of TL, FL, and BR. The straightening-based strategies still obviously outperformed the others when using all the mentioned models.

\section{Discussion}

In this paper, a novel multi-stage segmentation framework was constructed for extracting AD from CT images and divid- 
Table 2. TL, FL, and BR segmentation results of the 6-fold testing sets using end-to-end multi-class or multi-stage strategies. In each group, both the CNN 2 of NS and $S$ connected with the same $\mathrm{CNN}_{1}$, eliminating the influence of the $\mathrm{AO}$ segmented in the first stage. The BR results of NS and $\mathrm{S}$ were the same.

\begin{tabular}{|c|c|c|c|c|c|c|c|c|c|c|}
\hline \multirow[t]{2}{*}{ Networks } & \multirow[t]{2}{*}{ Strategies } & \multicolumn{3}{|l|}{ DSC } & \multicolumn{3}{|l|}{$\mathrm{MBD}(\mathrm{mm})$} & \multicolumn{3}{|l|}{$\mathrm{HD}_{95}(\mathrm{~mm})$} \\
\hline & & $\mathrm{TL}$ & FL & $\mathrm{BR}$ & $\mathrm{TL}$ & FL & BR & $\mathrm{TL}$ & FL & $\mathrm{BR}$ \\
\hline \multirow[t]{3}{*}{ 3D U-Net } & Multi-class & $0.92(0.04)$ & $0.90(0.18)$ & $0.88(0.03)$ & $1.34(0.99)$ & $5.09(35.47)$ & $1.37(1.16)$ & $4.92(4.12)$ & $8.11(32.18)$ & $4.78(5.55)$ \\
\hline & NS & $0.94(0.04)$ & $0.92(0.12)$ & $0.89(0.02)$ & $0.92(0.98)$ & $2.19(19.25)$ & $1.34(1.06)$ & $3.09(3.55)$ & $4.73(18.70)$ & $4.55(4.65)$ \\
\hline & $\mathrm{S}$ & $0.95(0.04)$ & $0.94(0.07)$ & - & $0.85(0.87)$ & $1.53(3.46)$ & - & $2.76(3.29)$ & $3.93(7.42)$ & - \\
\hline \multirow[t]{3}{*}{ SegNet } & Multi-class & $0.88(0.07)$ & $0.85(0.13)$ & $0.87(0.03)$ & $1.75(1.16)$ & $4.81(17.30)$ & $1.38(0.84)$ & $6.82(4.36)$ & $10.28(19.08)$ & $4.92(3.64)$ \\
\hline & NS & $0.92(0.06)$ & $0.90(0.10)$ & $0.87(0.02)$ & $1.09(0.87)$ & $2.11(9.09)$ & $1.30(0.66)$ & $3.97(3.57)$ & $5.18(10.30)$ & $4.38(3.02)$ \\
\hline & $\mathrm{S}$ & $0.96(0.02)$ & $0.95(0.04)$ & - & $0.57(0.38)$ & $0.84(1.70)$ & - & $1.85(1.75)$ & $2.28(4.74)$ & - \\
\hline \multirow[t]{3}{*}{ DeepLabV3+ } & Multi-class & $0.86(0.10)$ & $0.83(0.16)$ & $0.81(0.10)$ & $2.62(5.31)$ & $5.89(17.98)$ & $2.61(1.87)$ & $8.10(8.91)$ & $11.56(20.28)$ & $9.88(9.83)$ \\
\hline & NS & $0.85(0.13)$ & $0.80(0.22)$ & $0.81(0.13)$ & $2.71(3.58)$ & $4.72(15.50)$ & $2.32(1.80)$ & $7.67(7.25)$ & $9.89(16.44)$ & $9.19(9.33)$ \\
\hline & $\mathrm{S}$ & $0.93(0.07)$ & $0.89(0.17)$ & - & $1.43(2.06)$ & $3.15(11.30)$ & - & $4.60(6.81)$ & $7.03(17.33)$ & - \\
\hline \multirow[t]{3}{*}{ Ours } & Multi-class & $0.94(0.05)$ & $0.91(0.14)$ & $0.88(0.03)$ & $1.00(1.02)$ & $3.08(16.62)$ & $1.25(0.97)$ & $3.37(3.67)$ & $5.59(18.28)$ & $3.81(3.86)$ \\
\hline & NS & $0.94(0.05)$ & $0.92(0.12)$ & $0.89(0.03)$ & $0.86(0.85)$ & $2.38(16.52)$ & $1.21(0.96)$ & $2.75(3.25)$ & $4.04(14.21)$ & $3.58(4.13)$ \\
\hline & $\mathrm{S}$ & $0.96(0.03)$ & $0.95(0.06)$ & - & $0.55(0.45)$ & $0.69(1.16)$ & - & $1.72(1.83)$ & $2.05(4.46)$ & - \\
\hline
\end{tabular}

ed it into three different structures: TL, FL and BR. Two 3-D fully convolutional networks and an aorta straightening method were embedded in this framework, responsible for extracting the aortic features and simplifying the vascular complexity, respectively. By using strategy comparison, we demonstrated the advantage of the proposed framework and confirmed that the anatomy simplification using aorta straightening could significantly improve the accuracy of the dual-lumen segmentation.

We listed the results of the reported AD segmentation methods in Table 3 and divided them into two categories, i.e., the rule-based ones and those using DL. Unfortunately, these reported methods were evaluated with different datasets and evaluation metrics and there is no publicly available type $\mathrm{B} A D$ dataset that could support the comparison of the different models. However, we tried to train and test the DL-based framework proposed by Cao et al. (2019) with our dataset and the reported configurations, which also used a multi-stage design. As a result, our framework yielded obviously higher Dice scores on the testing set (i.e., 0.96 versus 0.86 for the AO, 0.92 versus 0.82 for TL, and 0.93 versus 0.84 for FL). As illustrated in section 3.1. this method resized the original image to a smaller size in the preprocessing and trained the network with the entire aorta, but the feature loss caused by the compression might adversely affect the segmentation performance.

The separation of TL and FL was the most challenging part in the $\mathrm{AD}$ segmentation task, because the start position and the length of dissections varied in different patients, and the morphology of the flap was invariably complex along the aorta. Moreover, the curved shape made the dual-lumen relative location more difficult to discern, although the aortic curvature and torsion were smaller and simpler comparing to small vessels like cerebral (Wolterink et al., 2016) and coronary arteries (Livne et al. . 2019). All these prior characteristics were nonnegligible obstacles that made learn the luminal continuity and dual-lumen differences difficult. By visualizing the 3-D reconstructions in Figure 7, the local fracture or shift of TL and FL were very common segmentation errors and obviously caused damage to luminal continuity. It was also the key bottleneck influencing the clinical and scientific practicality of the framework.

Aorta straightening brought significant improvements for the dual-lumen segmentation by efficiently alleviating the error above. Though adding the straightening step between the two networks made the segmentation not end-to-end, it was still greatly beneficial for this task because the straightened aorta had simpler and similar morphological characteristics in the transformed space. Moreover, based on the straightening, $\mathrm{CNN}_{2}$ could avoid paying attention to the features of curvature and torsion, which were saved directly in the centerline information. The relative location and the morphological difference of the dual lumen became the key knowledge learned by the network, which might effectively reduce the local luminal fractures or shifts.

Considering the limitation of the GPU memory in this work, it was impossible to directly train the network using the entire CT image with original resolution and large image size. We chose to use the multi-class subvolume segmentation strategy to approximate the end-to-end design, which also extrracting extracted TL, FL, and BR simultaneously but achieved unsatisfactory performance compared with the proposed framework. Though the end-to-end segmentation was more efficien$\mathrm{t}$, it could not learn the curvature and torsion features and the continuity of the single lumen well only based on insufficient dual-lumen contextual information of subvolumes, such that some local features could be considered as keys for judging the lumens. For example, the FL was usually larger than the TL locally, hence it might be easier to cause local shifts in region$\mathrm{s}$ where the volume and shape of the dual lumen were similar. The other end-to-end segmentation strategy was resizing the C$\mathrm{T}$ image to a smaller size but has been proven to be unfavorable for this task because of the severe feature loss.

By removing the straightening step, the strategy NS was a compromise using only two cascaded networks for the segmentation. It used $\mathrm{CNN}_{1}$ to learn the significant structural differences between the aortic trunk and branches, generating fewer outliers of AO and achieved slight advantage on BR (Figure 6) because the task was simplified into subtasks. We could find that TL and FL results were more compact than the ones of endto-end method, but the optimal values were significantly lower. It was understandable because the training of $\mathrm{CNN}_{2}$ with the entire aorta enlarged the receptive field and was conducive to learning the global vascular structure. However, the AO should be interpolated to a consistent size in the $\mathrm{Z}$ axis for the training, which could be harmful because the normalized resolution was 




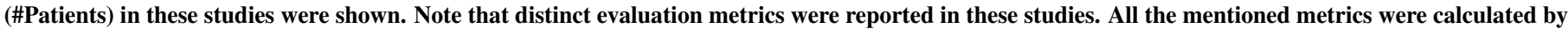
our framework for a more comprehensive comparison.

\begin{tabular}{|c|c|c|c|c|c|c|c|c|c|}
\hline \multirow[t]{2}{*}{ Type } & \multirow[t]{2}{*}{ Study } & \multirow[t]{2}{*}{ \#Patients } & \multicolumn{5}{|l|}{$\mathrm{AO}$} & \multirow{2}{*}{$\frac{\mathrm{TL}}{\mathrm{DSC}}$} & \multirow{2}{*}{$\frac{\mathrm{FL}}{\mathrm{DSC}}$} \\
\hline & & & DSC & $\operatorname{MBD}(\mathrm{mm})$ & Precision & Recall & Specificity & & \\
\hline \multirow{4}{*}{$\begin{array}{l}\text { Rule- } \\
\text { based }\end{array}$} & Kovács et al. (2006) & 17 & - & 1.71 & - & - & - & - & - \\
\hline & Lee et al. (2008) & 14 & - & - & - & 0.83 & 0.87 & - & - \\
\hline & Duan et al. (2016) & 10 & 0.93 & - & - & - & - & - & - \\
\hline & Fitria et al. (2019) & 11 & 0.94 & - & 0.96 & 0.92 & - & - & - \\
\hline \multirow[t]{2}{*}{ DL } & Cao et al. (2019) & 30 & 0.93 & - & - & - & - & 0.93 & 0.91 \\
\hline & Ours & 120 & 0.97 & 0.39 & 0.97 & 0.97 & 0.99 & 0.96 & 0.95 \\
\hline
\end{tabular}

lost and the essential curvature and torsion features might be destroyed. The straightening-based strategy just could simultaneously maintain the advantage of the full-image training and avoid the aortic feature loss by encoding the curvature and torsion information in the transformation procedure, further proving straightening was the core making the multi-stage designs perform better than the end-to-end ones. If conditions permit, the end-to-end training using the entire $\mathrm{AD}$ with high resolution might achieve better results like the multi-stage strategy NS, but could still be influenced by the complex morphological features.

This straightening method was designed based on the prior anatomy of the dissected aorta, because the extraction and the smoothing of the aortic centerline must be highly dependent on the natural tubular morphology of the vascular structure. Moreover, we considered that though the dual lumen were separated by the flap, the exterior outline of the aortic cross section could be still regarded as a circle, which meant it was suitable to approximately recover the original tubular shape of the aorta by filling the flap. In fact, tubular morphology was a common feature of vascular structures, and we believed this vessel straightening method and the segmentation pipeline could be also applied to other pathological vessels with complex structures and multiple scales, in particular, other dissected artery segmentation tasks. However, it might be not suitable for segmenting some lesions with aneurysms or thrombus (López-Linares et al. 2018), which caused relatively obvious damages to the local tubular shape of the blood vessels.

In Table 3, it was obvious that the straightening-based strategies still performed better than end-to-end multi-class ones even changing the network architecture, especially for the FL, whose shape and position were more complicated due to the thrombogenesis and might benefit more from the anatomy simplification. The network structure actually had a certain degree of influence on the segmentation. For instance, the multi-stage strategy NS had a tendency to improve the TL and FL results compared to the multi-class strategy. However, it was more obvious for SegNet but weaker for others, even appearing as a degradation for DeepLab model. This might be because some networks were more sensitive to the aortic feature loss during the image resizing for $\mathrm{CNN}_{2}$. Additionally, the results of BR were not significantly improved by the multi-stage strategies, but obviously showed the performance differences of these networks because of its complicated features. The branches are important vascular structures in hemodynamic analysis and the landmarks for morphological measurements. If the BR segmen- tation is not concerned in applications, we think the networks in the proposed straightening-based pipeline are replaceable.

The volumes of the dual lumen showed the capability of the framework for understanding the global AD anatomy, while the tears indicated the performance on grasping detailed local structures. For the clinical application, the accurate volumes might support quantitative evaluation of the long-term outcome of $\mathrm{AD}$ patients, while the shape and position of the tear could improve the surgical planning (Karmonik et al., 2010). We could further ensure that the TL was accurate enough in the local region where the primary tear occured, meaning that virtual intervention could be carried out (Chen et al., 2018a; Spranger et al. 2015) and potentially improved the success of the operation. However, it was difficult to automatically extract the tear because there was no intensity-based difference between the tear and the surrounding lumens. The variety of the flap shape at the aortic arch made it confusing to delineate the tears empirically. Fortunately, the straightening-based segmentation effectively simplified the flap shape so that achieved satisfactory performance on the recognition of the primary tears.

In future work, we will further evaluate the performance of the framework on the post-operative images and type A AD segmentation, while improving the performance on BR by modifying the subvolume cropping method and the loss function. Moreover, we will try to extract more morphological parameters of type $\mathrm{B} A D$ based on the segmentation, such as curvature and torsion of the aorta, position and area of the tear, and diameters of the dual lumen, which might greatly assist clinical diagnosis and treatment.

\section{Conclusion}

To conclude, this study constructed a novel DL-based type B AD segmentation framework in a multi-stage manner. Two cascaded CNNs and a novel aorta straightening method were embedded in the pipeline. The anatomy simplification of the $\mathrm{AD}$ based on the proposed aorta straightening was confirmed to be the core step significantly improving the TL and FL segmentation performances. Both the global volumetric features and local characteristics of primary tears could be well identified or quantified using the proposed framework. Moreover, this framework extracted all main branch vessels and potentially support the implementation of hemodynamic simulations. We believe we have shown that this framework may offer advantages over previous methods and may have great potential for clinical application. 


\section{Acknowledgments}

The authors would like to thank Shukun (Beijing) Network Technology Co., Ltd. for providing additional computing resources and technical support. This study was supported by the National Key R\&D Program of China (2018AAA0102600 and 2018YFC0115400), the National Natural Science Foundation of China (81970404, 81770465 and 81911530224), the Beijing Municipal Science \& Technology Commission (Z191100010618004), Beijing Natural Science Foundation (Z190014 and L192010), the Key R\&D Program of Zhejiang Province (2019C03013) and the Beijing Nova Program (Z181100006218008).

\section{Appendix A. Coefficient test for straightening}

A group of experiments was designed for evaluating the influence of the key coefficients in straightening on the final duallumen segmentation. In our design, we tried to change the order of magnitude for the scalars $\beta$ and $\omega$ in Eq. (5), i.e., from $10^{-2}$ to 10 , and performed the straightening using each pair of $\beta$ and $\omega$ based on the same 120 CT images. Totally 16 sets of straightened $\mathrm{AD}$ were generated. We used the same one fold in each set to train and evaluate $\mathrm{CNN}_{2}$ in the multi-stage pipeline and calculated DSC, MBD, and $\mathrm{HD}_{95}$ on the testing set. All the results of TL and FL were presented in Table A1 and Table A2, respectively. Two-sided and paired Wilcoxon signed rank test was used for testing the statistical difference between the configuration with $\beta=0.1, \omega=0.1$ (used in the current framework) and others $\left(p<5 \cdot 10^{-2}\right)$. We could find that there was no obvious trend shown in the comparisons. The used configuration $(\beta, \omega=0.1)$ just resulted in slightly higher dice score. No significant difference was found in any of these comparisons.

Table A1. Coefficient test for the influence of $\beta$ and $\omega$ in Eq.5 on the TL segmentation performance. Numbers with ${ }^{*}$ indicate significant difference compared to $\beta=0.1, \omega=0.1$.

\begin{tabular}{|c|c|c|c|c|}
\hline & $\omega=0.01$ & $\omega=0.1$ & $\omega=1$ & $\omega=10$ \\
\hline$\beta$ & \multicolumn{4}{|c|}{ DSC } \\
\hline 0.01 & $.951(.044)$ & $.949(.041)$ & $.952(.040)$ & $.949(.063)$ \\
\hline 0.1 & $.948(.051)$ & $.954(.036)$ & $.953(.037)$ & $.951(.044)$ \\
\hline 1 & $.952(.030)$ & $.951(.042)$ & $.946(.048)$ & $.948(.041)$ \\
\hline 10 & $.951(.040)$ & $.951(.040)$ & $.949(.041)$ & $.954(.032)$ \\
\hline$\beta$ & \multicolumn{4}{|c|}{$\operatorname{MBD}(\mathrm{mm})$} \\
\hline 0.01 & $0.55(0.41)$ & $0.57(0.41)$ & $0.57(0.41)$ & $0.59(0.48)$ \\
\hline 0.1 & $0.56(0.41)$ & $0.53(0.34)$ & $0.53(0.37)$ & $0.55(0.40)$ \\
\hline 1 & $0.53(0.30)$ & $0.54(0.38)$ & $0.57(0.46)$ & $0.54(0.37)$ \\
\hline 10 & $0.54(0.33)$ & $0.53(0.37)$ & $0.56(0.42)$ & $0.58(0.38)$ \\
\hline$\beta$ & \multicolumn{4}{|c|}{$\mathrm{HD}_{95}(\mathrm{~mm})$} \\
\hline 0.01 & $1.77(2.20)$ & $1.84(2.03)$ & $1.81(1.89)$ & $1.84(2.00)$ \\
\hline 0.1 & $1.80(2.11)$ & $1.74(1.83)$ & $1.80(2.06)$ & $1.74(1.88)$ \\
\hline 1 & $1.66(1.71)$ & $1.75(2.02)$ & $1.89(2.24)$ & $1.75(1.86)$ \\
\hline 10 & $1.71(1.80)$ & $1.81(2.06)$ & $1.72(1.78)$ & $1.83(1.86)$ \\
\hline
\end{tabular}

\section{References}

Anthimopoulos, M., Christodoulidis, S., Ebner, L., Christe, A., Mougiakakou, S., 2016. Lung pattern classification for interstitial lung diseases using a deep convolutional neural network. IEEE Trans. Med. Imag. 35(5), 12071216.
Table A2. Coefficient test for the influence of $\beta$ and $\omega$ in Eq. (5) on the FL segmentation performance. Numbers with ${ }^{*}$ indicate significant difference compared to $\beta=0.1, \omega=0.1$.

\begin{tabular}{|c|c|c|c|c|}
\hline & $\omega=0.01$ & $\omega=0.1$ & $\omega=1$ & $\omega=10$ \\
\hline$\beta$ & \multicolumn{4}{|c|}{ DSC } \\
\hline 0.01 & $.941(.087)$ & $.937(.083)$ & $.941(.081)$ & $.940(.074)$ \\
\hline 0.1 & $.939(.091)$ & $.943(.064)$ & $.942(.078)$ & $.941(.062)$ \\
\hline 1 & $.946(.065)$ & $.938(.090)$ & $.938(.083)$ & $.943(.064)$ \\
\hline 10 & $.944(.066)$ & $.941(.082)$ & $.943(.069)$ & $.944(.072)$ \\
\hline$\beta$ & \multicolumn{4}{|c|}{$\mathrm{MBD}(\mathrm{mm})$} \\
\hline 0.01 & $0.68(0.84)$ & $0.69(0.76)$ & $0.67(0.83)$ & $0.65(0.74)$ \\
\hline 0.1 & $0.68(0.79)$ & $0.62(0.60)$ & $0.63(0.80)$ & $0.61(0.66)$ \\
\hline 1 & $0.64(0.81)$ & $0.68(0.99)$ & $0.68(0.82)$ & $0.60(0.64)$ \\
\hline 10 & $0.62(0.70)$ & $0.66(0.82)$ & $0.66(0.80)$ & $0.65(0.83)$ \\
\hline$\beta$ & \multicolumn{4}{|c|}{$\mathrm{HD}_{95}(\mathrm{~mm})$} \\
\hline 0.01 & $1.93(3.07)$ & $2.00(2.98)$ & $1.98(2.99)$ & $2.01(2.83)$ \\
\hline 0.1 & $2.02(2.91)$ & $1.98(2.69)$ & $1.85(2.81)$ & $1.85(2.66)$ \\
\hline 1 & $1.87(2.49)$ & $2.05(3.55)$ & $1.94(2.93)$ & $1.85(2.63)$ \\
\hline 10 & $1.88(2.64)$ & $1.92(2.84)$ & $1.91(2.93)$ & $1.86(2.74)$ \\
\hline
\end{tabular}

Badrinarayanan, V., Kendall, A., Cipolla, R., 2017. SegNet: A deep convolutional encoder-decoder architecture for image segmentation. IEEE Trans. Pattern Anal. Mach. Intell. 39(12), 2481-2495.

Cao, L., Shi, R., Ge, Y., Xing, L., Zuo, P., Jia, Y., Liu, J., He, Y., Wang, X., Luan, S., Chai, X., Guo, W., 2019. Fully automatic segmentation of type B aortic dissection from CTA images enabled by deep learning. Eur. J. Radiol. 121, 108713.

Chen, D., Müller-Eschner, M., Kotelis, D., Böckler, D., Ventikos, Y., von Tengg-Kobligk, H., 2013. A longitudinal study of type-B aortic dissection and endovascular repair scenarios: computational analyses. Med. Eng. Phys. 35(9), 1321-1330.

Chen, D., Wei, J., Deng, Y., Xu, H., Li, Z., Meng, H., Han, X., Wang, Y., Wan, J., Yan, T., Xiong, J., Tang, X., 2018a. Virtual stenting with simplex mesh and mechanical contact analysis for real-time planning of thoracic endovascular aortic repair. Theranostics 8(20), 5758-5771.

Chen, L.C., Zhu, Y., Papandreou, G., Schroff, F., Adam, H., 2018b. Encoderdecoder with atrous separable convolution for semantic image segmentation, in: The European Conference on Computer Vision (ECCV), pp. 801-818.

Chen, X., Lu, Y., Bai, J., Yin, Y., Cao, K., Li, Y., Chen, H., Song, Q., Wu, J., 2018c. Train a 3D U-Net to segment cranial vasculature in CTA volume without manual annotation, in: 2018 IEEE 15th International Symposium on Biomedical Imaging (ISBI 2018), pp. 559-563.

Çiçek, Ö., Abdulkadir, A., Lienkamp, S., Brox, T., Ronneberger, O., 2016. 3D U-Net: learning dense volumetric segmentation from sparse annotation, in: Ourselin, S., Joskowicz, L., Sabuncu, M., Unal, G., Wells, W. (Eds.), Medical Image Computing and Computer-Assisted Intervention - MICCAI 2016, Springer. pp. 424-432.

Criado, F., 2011. Aortic dissection: a 250-year perspective. Tex. Heart Inst. J. 38(6), 694-700.

Dehghan, E., Wang, H., Syeda-Mahmood, T., 2017. Automatic detection of aortic dissection in contrast-enhanced CT, in: 2017 IEEE 14th International Symposium on Biomedical Imaging (ISBI 2017), pp. 557-560.

Duan, X., Shi, M., Wang, J., Zhao, H., Chen, D., 2016. Segmentation of the aortic dissection from CT images based on spatial continuity prior model, in: 2016 8th International Conference on Information Technology in Medicine and Education (ITME), pp. 275-280.

Fetnaci, N., Łubniewski, P., Miguel, B., Lohou, C., 2013. 3D segmentation of the true and false lumens on CT aortic dissection images, in: Proc. SPIE Int. Soc. Opt. Eng. 8650. doi 10.1117/12.2003345

Fitria, M., Adriman, R., Morariu, A., Pauli, J., 2019. Comparison of denoising methods applied to CTA images of 3D segmentation of aortic dissection, in: 2019 IEEE International Conference on Cybernetics and Computational Intelligence (CyberneticsCom), pp. 92-97.

Frangi, A.F., Niessen, W.J., Vincken, K., Viergever, M., 2000. Multiscale vessel enhancement filtering, in: Wells, W.M., Colchester, A., Delp, S. (Eds.), Medical Image Computing and Computer-Assisted Intervention - MICCAI'98, pp. 130-137.

Gibson, E., Giganti, F., Hu, Y., Bonmati, E., Bandula, S., Gurusamy, K., Davidson, B., Pereira, S., Clarkson, M., Barratt, D., 2018. Automatic multi-organ 
segmentation on abdominal CT with dense V-networks. IEEE Trans. Med. Imag. 37(8), 1822-1833.

Hagan, P., Nienaber, C., Isselbacher, E., Bruckman, D., Karavite, D., Russman, P., Evangelista, A., Fattori, R., Suzuki, T., Oh, J., Moore, A., Malouf, J., Pape, L., Gaca, C., Sechtem, U., Lenferink, S., Deutsch, H., Diedrichs, H., Robles, J., Eagle, K., 2000. The international registry of acute aortic dissection (IRAD): new insights into an old disease. JAMA 283(7), 897-903.

He, T., Hu, J., Song, Y., Guo, J., Yi, Z., 2020. Multi-task learning for the segmentation of organs at risk with label dependence. Med. Image Anal. 61, 101666.

Hou, L., Samaras, D., Kurc, T., Gao, Y., Davis, J., Saltz, J., 2016. Patchbased convolutional neural network for whole slide tissue image classification, in: 2016 IEEE Conference on Computer Vision and Pattern Recognition (CVPR), pp. 2424-2433.

Huang, G., Liu, Z., van der Maaten, L., Weinberger, K., 2017. Densely connected convolutional networks, in: 2017 IEEE Conference on Computer Vision and Pattern Recognition (CVPR), pp. 2261-2269.

Imai, H., Matzek, S., Le, T., Negishi, Y., Kawachiya, K., 2018. Fast and accurate 3D medical image segmentation with data-swapping method. arXiv: 1812.07816

Karmonik, C., Bismuth, J., Redel, T., Anaya-Ayala, J., Davies, M., Shah, D., Lumsden, A., 2010. Impact of tear location on hemodynamics in a type B aortic dissection investigated with computational fluid dynamics, in: 2010 Annual International Conference of the IEEE Engineering in Medicine and Biology, pp. 3138-3141.

Khan, I.A., Nair, C.K., 2002. Clinical, diagnostic, and management perspectives of aortic dissection. Chest 122(1), 311-328.

Khened, M., Kollerathu, V., Krishnamurthi, G., 2018. Fully convolutional multi-scale residual denseNets for cardiac segmentation and automated cardiac diagnosis using ensemble of classifiers. Med. Image Anal. 51, 21-45.

Kovács, T., Cattin, P., Alkadhi, H., Wildermuth, S., Székely, G., 2006. Automatic segmentation of the aortic dissection membrane from 3D CTA images, in: Medical Imaging and Augmented Reality, Springer. pp. 317-324.

Krissian, K., Carreira, J., Monreal, J., Maynar, M., 2013. Semi-automatic segmentation and detection of aorta dissection wall in MDCT angiography. Med. Image Anal. 18(1), 83-102.

LeCun, Y., Boser, B.E., Denker, J.S., Henderson, D., Howard, R.E., Hubbard, W.E., Jackel, L.D., 1990. Handwritten digit recognition with a backpropagation network, in: Touretzky, D.S. (Ed.), Advances in Neural Information Processing Systems 2. Morgan-Kaufmann, pp. 396-404.

Lee, N., Tek, H., Laine, A., 2008. True-false lumen segmentation of aortic dissection using multi-scale wavelet analysis and generative-discriminative model matching, in: Proc. SPIE 6915. doi 10.1117/12.770610

Lee, T.C., Kashyap, R.L., Chu, C.N., 1994. Building skeleton models via 3D medial surface axis thinning algorithms. CVGIP Graph. Model. Image Process. 56(6), 462-478.

Livne, M., Rieger, J., Aydin, O., Taha, A.A., Akay, E., Kossen, T., Sobesky, J., Kelleher, J., Hildebrand, K., Frey, D., Madai, V., 2019. A U-Net deep learning framework for high performance vessel segmentation in patients with cerebrovascular disease. Front. Neurosci. 13.

López-Linares, K., Aranjuelo, N., Kabongo, L., Maclair, G., Lete, N., Ceresa, M., García-Familiar, A., Macía, I., Ballester, M.A.G., 2018. Fully automatic detection and segmentation of abdominal aortic thrombus in post-operative CTA images using deep convolutional neural networks. Med. Image Anal. 46, 202-214.

Lu, D., Heisler, M., Lee, S., Ding, W., Navajas, E., Sarunic, M., Beg, M.F., 2019. Deep-learning based multiclass retinal fluid segmentation and detection in optical coherence tomography images using a fully convolutional neural network. Med. Image Anal. 54, 100-110.

Maas, A., Hannun, A., Ng, A.Y., 2013. Rectifier nonlinearities improve neural network acoustic models, in: ICML Workshop on Deep Learning for Audio, Speech and Language Processing.

Morais, P., Vilaça, J., Queirós, S., Bourier, F., Deisenhofer, I., Tavares, J., D'hooge, J., 2017. A competitive strategy for atrial and aortic tract segmentation based on deformable models. Med. Image Anal. 42, 102-116.

Morariu, A., Zohourian, F., Dohle, D., Tsagakis, K., Pauli, J., 2016. Unsupervised extraction of the aortic dissection membrane based on a multiscale piecewise ridge model, in: 2016 IEEE 13th International Symposium on Biomedical Imaging (ISBI 2016), pp. 1368-1371.

Nazeri, K., Aminpour, A., Ebrahimi, M., 2018. Two-stage convolutional neural network for breast cancer histology image classification, in: Campilho, A., Karray, F., ter Haar Romeny, B. (Eds.), Image Analysis and Recognition,
Springer. pp. 717-726.

Nienaber, C.A., Clough, R.E., 2015. Management of acute aortic dissection. Lancet 385, 800-811.

Noothout, J., De Vos, B., Wolterink, J., Isgum, I., 2018. Automatic segmentation of thoracic aorta segments in low-dose chest CT, in: Proc. SPIE 10574. doi $10.1117 / 12.2293114$

Paszke, A., Gross, S., Chintala, S., Chanan, G., Yang, E., DeVito, Z., Lin, Z., Desmaison, A., Antiga, L., Lerer, A., 2017. Automatic differentiation in pytorch .

Rota Bulò, S., Porzi, L., Kontschieder, P., 2018. In-place activated batchnorm for memory-optimized training of DNNs, in: 2018 IEEE/CVF Conference on Computer Vision and Pattern Recognition (CVPR), pp. 5639-5647.

Shelhamer, E., Long, J., Darrell, T., 2016. Fully convolutional networks for semantic segmentation. IEEE Trans. Pattern Anal. Mach. Intell. 39(4), 640651 .

Spranger, K., Capelli, C., Bosi, G., Schievano, S., Ventikos, Y., 2015. Comparison and calibration of a real-time virtual stenting algorithm using finite element analysis and genetic algorithms. Comput. Methods Appl. Mech. Eng. 293, 462-480.

Trullo, R., Petitjean, C., Ruan, S., Dubray, B., Nie, D., Shen, D., 2017. Segmentation of organs at risk in thoracic CT images using a SharpMask architecture and conditional random fields, in: 2017 IEEE 14th International Symposium on Biomedical Imaging (ISBI 2017), pp. 1003-1006.

Tsai, T.T., Trimarchi, S., Nienaber, C.A., 2009. Acute aortic dissection: perspectives from the international registry of acute aortic dissection (IRAD). Eur. J. Vasc. Endovasc. Surg. 37(2), 149-159.

White, A., Broder, J., Mando-Vandrick, J., Wendell, J., Crowe, J., 2013. Acute aortic emergencies-part 2: aortic dissections. Adv. Emerg. Nurs. J. 35(1), $28-52$.

Wolterink, J., Leiner, T., De Vos, B., van Hamersvelt, R., Viergever, M., Isgum, I., 2016. Automatic coronary artery calcium scoring in cardiac CT angiography using paired convolutional neural networks. Med. Image Anal. 34(1), $123-136$.

Xu, H., Xiong, J., Han, X., Mei, Y., Shi, Y., Wang, D., Zhang, M., Chen, D., 2020. Computed tomography based hemodynamic index for aortic dissection. J. Thorac. Cardiovasc. Surg. doi https://doi.org/10.1016/j. jtcvs.2020.02.034

Yang, X., Bian, C., Yu, L., Ni, D., Heng, P., 2018. Hybrid loss guided convolutional networks for whole heart parsing, in: Statistical Atlases and Computational Models of the Heart. ACDC and MMWHS Challenges (STACOM), Springer. pp. 215-223.

Zreik, M., van Hamersvelt, R., Wolterink, J., Leiner, T., Viergever, M., Isgum, I., 2018. A recurrent CNN for automatic detection and classification of coronary artery plaque and stenosis in coronary CT angiography. IEEE Trans. Med. Imag. 38(7), 1588-1598. 